Published in final edited form as:

J Crit Care. 2013 December ; 28(6): . doi:10.1016/j.jcrc.2013.06.021.

\title{
The relationship between positive end expiratory pressure and cardiac index in patients with acute respiratory distress syndrome
}

\author{
Wassim H Fares, MD MSc ${ }^{1}$, Shannon S Carson, MD $^{2}$, and for the NIH NHLBI ARDS Network \\ ${ }^{1}$ Yale University, School of Medicine, Department of Internal Medicine, Section of Pulmonary, \\ Critical Care \& Sleep Medicine, New Haven, CT 06510 \\ ${ }^{2}$ University of North Carolina, School of Medicine, Department of Medicine, Section of Pulmonary \\ and Critical Care Medicine, Chapel Hill, NC 27599
}

\begin{abstract}
Purpose-To evaluate the association between positive end-expiratory pressure (PEEP) and cardiac index in patients with acute respiratory distress syndrome (ARDS).

Methods-This is a secondary cross-sectional analysis of the FACTT multi-center randomized controlled trial enrolling adult patients within 48 hours of ARDS onset. Patients randomized to the pulmonary artery catheter arm, who had PEEP and cardiac index measurements performed within a short period of each other during the first 3 days of the FACTT study enrollment were included in this study. Since FACTT had a $2 \times 2$ factorial design, half of the patients were in a 'liberal fluids' study arm, and the other half were in a 'conservative fluids' study arm.
\end{abstract}

Results-The final study population (833 measurements or observations, in 367 patients) was comparable to the original overall FACTT study population. The mean PEEP level used was $8.2 \pm$ $3.4 \mathrm{~cm} \mathrm{H}_{2} \mathrm{O}$, and the mean cardiac index was $4.2 \pm 1.2 \mathrm{liters} / \mathrm{minute} / \mathrm{m}^{2}$. There was no association between PEEP and cardiac index in patients with ARDS, even when adjusted for APACHE score, age, fluid study arm in FACTT, and sepsis.

Conclusion-In patients with ARDS who are managed with liberal or conservative fluid management protocols, PEEP is not associated with lower cardiac index.

\section{Keywords}

Mechanical ventilation; ARDS; cardiac function; cardiac index; heart-lung interaction

\section{Introduction}

Acute respiratory distress syndrome (ARDS) has a 25\% - 45\% two-month [1-4] and >60\% one-year mortality [5]. The estimated incidence of ARDS in the US is about 200,000 cases /

(C) 2013 Elsevier Inc. All rights reserved.

Corresponding Author: Wassim H Fares, MD MSc, Yale University, 15 York Street, LCI 105-, New Haven, CT 06510, Tel: (203) 785-4196, Fax: (203) 785-3634, wassim.fares@yale.edu.

Publisher's Disclaimer: This is a PDF file of an unedited manuscript that has been accepted for publication. As a service to our customers we are providing this early version of the manuscript. The manuscript will undergo copyediting, typesetting, and review of the resulting proof before it is published in its final citable form. Please note that during the production process errors may be discovered which could affect the content, and all legal disclaimers that apply to the journal pertain.

Conflicts of interest

The authors report no conflicts of interest with regard to this manuscript. 
year [1,2]. ARDS has multiple contributing pathophysiologic factors, including vasoconstriction, endothelial dysfunction, myointimal proliferation, highly inflammatory milieu, and micro-thrombi [6]. Since ventilation-induced lung injury including barotrauma, volutrauma, and biotrauma [7] worsen all of the above processes, one mechanism by which to improve survival in ARDS patients is to use different ventilatory strategies to decrease the risk of ventilation-induced lung injury [8]. Relatively higher positive end-expiratory pressure (PEEP) levels $\left(\sim 15 \mathrm{~cm} \mathrm{H}_{2} 0\right)$ theoretically decrease the risk of biotrauma by preventing de-recruitment of alveoli and optimizing oxygenation. However, since the pathologic pulmonary lesion in ARDS is heterogeneous, PEEP might induce alveolar recruitment in certain segments of the lungs, while simultaneously causing over-distension in other segments.

The optimal PEEP for patients with ARDS is not known, and there is conflicting evidence in the literature related to the relationship between PEEP and cardiac function [9-16]. PEEP potentially recruits alveoli and improves functional residual capacity (FRC) $[17,18]$. However, clinicians are concerned about compromising cardiac index with high PEEP. Such an effect is believed to be due to increased intra-thoracic pressure exerted by the PEEP. The PEEP-induced increase in alveolar pressure could increase right ventricular (RV) afterload, and right atrial pressure which could decrease venous return / RV preload. Based on these phenomena, in a compliant lung, both of these PEEP-induced effects (increased RV afterload and decreased RV preload) have been shown to decrease cardiac output. It is possible though that the effect of high PEEP on cardiac index is attenuated by the significant pulmonary edema and decreased lung compliance that is characteristic of the ARDS patient population.

A post-hoc sub-group analysis of individual data of 3 major randomized controlled clinical trials [19-22] showed an association between improved in-hospital survival in largely unselected moderate to severe ARDS patients with higher PEEP (mean $15 \mathrm{~cm}$ ) versus lower PEEP (mean $9 \mathrm{~cm}$ ) [34\% versus 39\%; relative risk $(\mathrm{RR})=0.90 ; \mathrm{p}=.049 ; \mathrm{n} \sim 1,900$ ]. Despite the possible improvement in outcome associated with higher PEEP in moderate to severe ARDS, many clinicians remain reluctant to use higher PEEP levels because of the presumed hemodynamic effects.

We sought to determine the relationship between PEEP and cardiac index in patients with ARDS using a retrospective cross-sectional study design. We hypothesized that increased PEEP levels are not associated with lower cardiac index in patients with moderate to severe ARDS in patients managed on a fluid protocol.

\section{Methods}

This study is a secondary analysis of the United States National Institute of Health (NIH), National Heart Lung and Blood Institute (NHLBI), Acute Respiratory Distress Syndrome Clinical Trials Network (ARDSnet) Fluid and Catheter Treatment Trial (FACTT) [23]. In brief review, FACTT study used a $2 \times 2$ factorial design randomizing approximately 1,000 ARDS patients (in 20 centers across the US, some of which included more than one hospital) into two groups of: 1) liberal versus conservative fluid management, and 2) pulmonary artery catheter (PAC) versus central venous catheter placement [3, 23]. The protocol of the FACTT study has been previously published [3]. To briefly summarize the population studied as it relates to this study, the major inclusion criterion was an acute lung injury diagnosis (as defined by The American - European Consensus Conference on ARDS) [24] of $<48$ hours in duration. Major exclusion criteria to note in FACTT were mostly select chronic conditions that could independently influence survival (e.g., expected 6-month survival $<50 \%$ ) and / or ventilator weaning. Patients who were enrolled in the trial were 
randomized to liberal or conservative fluid protocols that directed fluid management including fluid boluses in response to hypotension, or diuresis when hemodynamically stable to achieve pre-selected goals in central venous pressure (CVP) or pulmonary artery occlusion pressure (PAOP). The protocols also included instruction for use of inotropes in patients with a low cardiac index.

The de-identified study population is drawn from the patients in the PAC arm of the FACTT study that actually had a PAC placed (513 were randomized to the PAC arm of which 501 actually received it). For the primary hypothesis, only observations with $\mathrm{PaO} 2 / \mathrm{FiO} 2(\mathrm{PF})$ $\varsigma 200$ are included. Moderate to severe ARDS patients are solely included in the primary study question based on the data presented in the meta-analysis that showed association with benefit of higher PEEP in this subgroup [22]. Patients with PF of $>200$ are included in a secondary analysis.

This study is an observational non-interventional study. The primary exposure or independent variable is the PEEP, and the outcome or dependent variable is the cardiac index. For the primary analysis, only data from the first 3 days of mechanical ventilation were included because after day 3 of enrollment, the FACTT protocol gave the option to replace the PAC by a central venous catheter if hemodynamic stability was maintained [3]. This in theory could have led to variation in patient care from day 4 and onward with a biased selection of patients having a PAC during that period of time, i.e., beyond the first 3 days of study enrollment. The institutional review board (IRB) at the University of North Carolina at Chapel Hill approved this secondary analysis study (IRB\# 11-0762), and waiver of consent was granted.

\section{Statistical Considerations}

Each day's measurement was the unit of analysis (observation). Non-independence of observations within patients may result in variance estimates that are too small. To avoid such bias, the analysis was done using generalized estimating equations (GEE) which apply a robust estimator of variance to adjust for intra-class correlation (ICC) due to the nonindependence of observations [25,26]. The sample size estimates have taken this adjustment into account. Since a large number of observations (see results) were not accounted for using GEE analysis when there was no more than 1 observation / patient, the same analysis was done using cluster analysis without GEE to maximize the number of observations included. As noted below, the cluster analysis results were not different from the GEE analysis results.

\section{Analytic Plan}

Sample descriptive data were expressed as means \pm standard deviation for normally distributed continuous variables and as counts / percentages for categorical variables. We used a 2-sample student t-test to compare the means of continuous variables between 2 groups. Bivariate relationships between continuous variables (e.g., PEEP and cardiac index) were evaluated by the Pearson's correlation coefficient (r).

For the primary analysis, simple and multiple linear regression models were performed. As mentioned above, the primary outcome variable of this study is cardiac index, and the primary exposure variable is PEEP as a continuous variable. The analysis was also run using PEEP as a dichotomous variable ( $<12$ versus $\geq 12 \mathrm{~cm}$ ), which was also decided a priori. This PEEP cut-point of $12 \mathrm{~cm}$ was largely based on personal anecdotal experiences as the threshold for many clinicians' 'comfort level'.

The final mean cardiac index estimates were adjusted for the following potential confounding covariates: APACHE III score, age, FACTT study arm (liberal versus 
conservative fluids arm), and sepsis (present versus absent). Assessment for effect measure modification between PEEP and the other variables, i.e., interaction, was made first before evaluating confounding. In a sensitivity analysis, a model similar to the above model was run without adjusting for age since age is an important component of APACHE III that is already included in the model.

Since PEEP itself can affect the central venous pressure measurement, we did not adjust for the central venous pressure (CVP) due to expected colinearity. Additionally, increased intrathoracic pressure caused by the higher PEEP would also decrease cardiac index by decreasing venous return, i.e., decreased preload, making CVP part of the causal pathway. There has also been significant controversy about the accuracy of CVP as a measure of intravascular volume [27, 28].

In addition, the increased intra-thoracic pressure caused by PEEP could lead to higher measured hemodynamic pressures including the pulmonary capillary wedge pressure that might potentially impact cardiac function through increased RV afterload. This would also position the pulmonary capillary wedge pressure in the causal pathway of PEEP's effect on cardiac index, and thus we did not adjust for the wedge pressure. We decided a priori to use FACTT study arm (liberal versus conservative fluids arm) instead of fluid volume received in the primary multiple regression model because the study arm would encompass within it multiple other interventions (which include but are beyond just fluid volume) that could potentially affect cardiac index.

A 2-sided alpha of 0.05 was considered statistically significant for all tests for effect modification, and for the final adjusted mean cardiac index estimate comparison between PEEP categories, without adjusting for multiple testing. All statistical analyses were performed using STATA software version 11 IC for Windows (STATACorp, College Station, Texas; 2010).

\section{Sample Size calculation}

To our knowledge, the FACTT database is the largest available database with concurrent PEEP and cardiac index measurements in ARDS patients. We included all data points during the first 3 days of enrollment (potentially multiple readings for each patient). Based on what had been published on the FACTT study data and taking into account the difference in the size of each group [3,26] (Appendix E1), we made the following assumptions and expectations prior to obtaining the FACTT raw data:

Expected cardiac index mean in the low PEEP $(<12 \mathrm{~cm})$ group $=4.1 \pm 1.4 \mathrm{liters} / \mathrm{minute} / \mathrm{m}^{2}$. Expected cardiac index mean in the high PEEP $(\geq 12 \mathrm{~cm})$ group $=3.6 \pm 1.8$ liters $/$ minute $/ \mathrm{m}^{2}$. Based on these assumptions and taking into account an intra-class correlation $(1-3$ observations / patient), the total number of observations needed would be 735 (with a minimum of 368 patients): 148 observations in the high PEEP group and 587 in the low PEEP group with $80 \%$ power and a 2-sided alpha of 0.05 (Appendix E1).

\section{Results}

The study population comprised of 833 measurements in 367 patients (Fig. 1). The distribution of the characteristics of the 367 patients (Table 1) was similar to the original overall FACTT cohort [3]. Out of the 833 observations, 556 had PF $\leq 200$ (among 287 patients). Seventy-nine patients had one complete observation only, 110 patients had 2 complete observations, and 178 patients had 3 complete observations. The average PEEP level was $8.3 \pm 3.4 \mathrm{~cm}$ and the average cardiac index was $4.2 \pm 1.2 \mathrm{liters} / \mathrm{minute} / \mathrm{m}^{2}$ (Fig. 2 $\&$ Table 2). There was no correlation between PEEP and cardiac index $(r=0.05 ; \mathrm{p}=0.17)$. 
This unadjusted PEEP / cardiac index relationship was not different between those with a PF $\leq 200$ versus those with a PF $>200$.

When adjusted for APACHE III score, age, FACTT study arm (liberal versus conservative fluids arm), and sepsis (present versus absent), there was no statistically significant relationship between PEEP and cardiac index for those with PF $\leq 200$ (p=0.71) (Fig. 3). In a post-hoc analysis, static lung compliance was decided a posteriori (subsequent to suggestion by experts in the field) to be adjusted for in addition to the above mentioned co-variables in the regression model, and it did not change the relationship between PEEP and cardiac index $(p=0.73)$ (Appendix E2). Similarly, there was no statistically significant relationship between PEEP and cardiac index in the subgroup with PF > 200 or in the whole study population of 833 observations. These results were also not different with PEEP dichotomized: $\mathrm{PEEP}<12 \mathrm{~cm} \mathrm{H}_{2} \mathrm{O}\left[\mathrm{n}=703\right.$, mean cardiac index $\left.=4.2 \mathrm{liters} / \mathrm{minute} / \mathrm{m}^{2}\right]$ and PEEP $\geq 12 \mathrm{~cm} \mathrm{H}_{2} \mathrm{O}\left[\mathrm{n}=130\right.$, mean cardiac index $=4.3$ liters $/$ minute $\left./ \mathrm{m}^{2}\right](\mathrm{p}=0.17)$. This lack of association between the dichotomized PEEP groups and the cardiac index persisted even when adjusting for APACHE III score, FACTT study arm (liberal versus conservative fluids arm), and sepsis ( $\mathrm{p}=0.49)$.

Using GEE $(n=408 ; p=0.8)$ or cluster analyses $(n=552 ; p=0.8)$, there was no significant association between PEEP and cardiac index in the patients with moderate to severe ARDS, when adjusted for the same variables (APACHE III score, age, FACTT fluids study arm, and sepsis) (a priori decided co-variables). Similar results were noted when also adjusted for static pulmonary compliance (post-hoc analysis). Patients without moderate or severe ARDS showed comparable lack of association between PEEP and cardiac index. Other post-hoc sensitivity analyses were done and are shown in Appendix E3, including observations beyond day 3 of FACTT study recruitment, patients who were hemodynamically unstable (i.e., on vasopressors), and with adjusting for actual fluids given (rather than a dichotomous FACTT study arm assignment). All of these analyses consistently showed lack of association between PEEP and cardiac index.

\section{Discussion}

These results suggest that there is no significant relationship between PEEP levels and cardiac index in patients with moderate to severe ARDS who are managed on a fluid and hemodynamic protocol. Patients within $1-5$ days of ARDS onset that received mechanical ventilation with high PEEP levels did not have lower cardiac index compared to patients that received mechanical ventilation with low PEEP levels. This finding could encourage in this patient population more use of higher PEEP levels which seem to have multiple clinical advantages. This study is the largest of its kind to look at the association between PEEP and cardiac index in ARDS patients in the current era of protective lung ventilation in ARDS management.

The benefits of PEEP are multiple in patients with ARDS. PEEP potentially increases the functional residual capacity (FRC), causes alveolar recruitment, pushes water out of the alveolar vessels interstitium into the extra-alveolar vessels interstitium [17], and thus enhances ventilation with resultant improved ventilation - perfusion matching [18]. Another finding that supports the potential benefits of a relatively higher PEEP level is that the transpulmonary pressure is often negative and the respiratory system compliance is often not optimal when following the ARDSnet standard of care recommendations [29].

The major disadvantage is the risk of barotrauma and a potential effect on central hemodynamics via increasing intra-thoracic pressure which encompasses alveolar, pleural, and juxta-cardiac pressures. The distribution of alveoli recruitment / over-distension 
pressures impacts the benefit / risk ratio of high PEEP levels which is also dependent on the severity of ARDS and the extent and distribution of lung damage. Lungs with a diffuse and homogeneous ARDS pattern might not get as much patchy alveoli over-distension as lungs with mild ARDS or lobar ARDS pattern [30]. The functional lung volume loss, which is associated with the severity of lung injury, theoretically decreases the likelihood of alveolar over-distension in these de-recruited units. This functional lung volume loss also decreases the transmittance of intrathoracic pressure to the thoracic vasculature.

The PEEP level for an optimal cardiac index is not known in patients with ARDS [31]. Many clinicians are hesitant to increase PEEP in ARDS patients to $>10-12 \mathrm{~cm}$, mostly because of concerns about compromising cardiac index. However this approach is not supported by strong or consistent evidence in ARDS [32,33]. As mentioned above, the meta-analysis of individual data of 3 major randomized controlled trials showed improved in-hospital survival in moderate to severe ARDS patients with higher PEEP (mean $15 \mathrm{~cm}$ ) versus lower PEEP (mean $9 \mathrm{~cm}$ ) [19-22]. The first of the 3 above mentioned trials, the ALVEOLI trial, included 550 patients with PF $\leq 300$, and it was stopped for perceived futility [19]. The LOVS trial included almost 1,000 patients with PF $\leq 250$ [20], while the EXPRESS trial included 768 patients with PF $\leq 300$ [21]. Unfortunately, these trials did not have hemodynamic measurements to assess the effect of PEEP on cardiac index, so it is not clear whether the possible improved survival of moderate to severe ARDS patients and worsened survival in mild ARDS patients on the higher PEEP levels are due to differential effects of PEEP on cardiac index.

There is a paucity of trials, with conflicting results, that looked at the direct effect of varying levels of PEEP on cardiac index in patients with ARDS. A study with 12 patients with ARDS showed that cardiac output did not decrease on PEEP of $20 \mathrm{~cm}$ and pressure control of $30 \mathrm{~cm}$ (for a peak and plateau inspiratory pressures of about $50 \mathrm{~cm}$ ) [10]. A French group that was primarily looking at the extent of alveolar recruitment using computed tomography (CT) scans found a significant decrease in cardiac index from 4.6 to 3.5 liters $/ \mathrm{minute} / \mathrm{m}^{2}$ $(\mathrm{p}=0.005)$ without much change in heart rate (102 versus $98 / \mathrm{min})$ upon increasing PEEP from $0 \mathrm{~cm}$ to $15 \mathrm{~cm}$ [11]. The mean systemic arterial pressure dropped from 94 to $78 \mathrm{mmHg}$ $(\mathrm{p}=0.03)$ and mean pulmonary artery pressure stayed the same [11].

Schmitt et al also demonstrated increased right ventricular outflow impedance evaluated by Doppler echocardiography without heart catheterization at higher PEEP levels (13 versus 6 $\mathrm{cm}$ ) in 16 ARDS patients [12]. However the patients on the higher PEEP levels had better oxygenation which admittedly is not necessarily a criterion of patient improvement [8] but is one positive clinical marker. Mekontso et al looked at 11 patients with ARDS (PF $<150$ $\mathrm{mmHg}$ ) and showed improved oxygenation on PEEP levels of $10-11 \mathrm{~cm}$ compared to PEEP of about $5 \mathrm{~cm}$, however with significantly higher PaCO2 (71-75 versus $52 \mathrm{mmHg}$ ) [14]. The hyper-capnea was due to a lower tidal volume as planned per study design: 350 versus $550 \mathrm{ml}$ in the high versus low PEEP groups respectively to achieve a plateau pressure of $<30 \mathrm{~cm}$. The investigators attributed the drop in cardiac index (1.88 versus 2.6 liters/ minute $/ \mathrm{m}^{2}$ ) to this respiratory acidosis [14].

Another French group saw a 13\% decrease in cardiac index with increasing PEEP from 5 to $13 \mathrm{~cm}$ in 21 ARDS patients, and that drop was corrected with passive leg raising [15]. The trans-pulmonary gradient was elevated at the higher PEEP level, and the authors attributed the cardiac output restoration to decreased right ventricular afterload by recruiting collapsed pulmonary micro-vessels induced by increased central blood volume with passive leg raising [15]. Increasing PEEP from 9 to $14 \mathrm{~cm}$ in 85 ARDS patients without patent foramen ovale (PFO) shunting induced PFO shunting in 8 patients (9\%) [13]. Titration of PEEP from 5 to 10 then to $15 \mathrm{~cm}$ improved oxygenation in ARDS patients without PFO shunting $(\mathrm{N}=15)$ but 
did not have an effect on oxygenation in 21 patients with moderate-to-large PFO shunting [13].

Older reports have also suggested high enough PEEP to cause alveolar recruitment would actually decrease pulmonary artery pressures without a significant effect on cardiac output [34], and more recent reports have started looking at the differential effect of lung compliance levels on central hemodynamics measurements in ARDS patients [35]. Effective alveolar recruitment is typically accompanied by pulmonary capillary recruitment which would in turn improve central hemodynamics. Lower PEEP levels may not achieve such recruitment adequately. In one series, it was the plateau pressure that was strongly associated with elevated pulmonary artery pressures [36]. Although not tested in this study, an individualized strategy to maximize the PEEP level while attempting to stay within acceptable plateau levels, typically $<28-30 \mathrm{~cm}$, might make the most sense in these patients.

These studies [9-16, 31] highlight the lack of consistency or agreement in the literature regarding the association between PEEP and cardiac index in patients with ARDS. These results showing no association could be explained by decreased lung compliance in ARDS and thus decreased intra-thoracic pressure transmittance to the central chest vasculature. But it could also be attributed to adjustments made by clinicians in response to hemodynamic changes that occur with increases in PEEP. The FACTT study had specific guiding rules for fluids administration and / or inotropes. Such clinicians' responses may include decreasing diuresis, adjusting fluid volume, and /or initiation of vasopressors to restore hemodynamics. These responses could mitigate any effects PEEP might have on cardiac function. For the primary analysis, patients who were on vasopressors at any given time on the day that the PEEP and cardiac index measurements were done were a priori excluded. Finally, measures of static respiratory system compliance were adjusted for. However since minute to minute measurements were not recorded, this analysis may not be sensitive enough to differentiate between lung compliance and hemodynamic management as possible explanations for lack of association between PEEP and cardiac index in moderate to severe ARDS.

There might have been other confounding variables related to PEEP and cardiac index that were not accounted for. Not all factors that could potentially affect cardiac index were routinely measured such as tricuspid regurgitation or changes in right ventricle dimensions, or are practically measurable such as the extent of transmission of the PEEP-induced intrathoracic pressure to the right heart chambers or inferior vena cava. In addition, cardiac index measurements have an inherent range of error, because of both technical aspects and theoretical assumptions which are the basis of these measurements [37], and which may not necessarily apply consistently to patients on mechanical ventilation.

\section{Conclusions}

This cross-sectional analysis of the FACTT data did not find an association between PEEP and cardiac index in patients with ARDS who are managed on a fluid and hemodynamic protocol in the post lung protective ventilation era. Individualized therapy to maximize recruitment using higher PEEP levels on top of lung protective ventilation and maintaining low plateau pressures is a reasonable approach to care, although some compensation for hemodynamic changes may be necessary. This observation is clinically relevant because of the suggestion, though not yet proven, of better outcomes in moderate to severe ARDS patients with higher PEEP levels by one recent meta-analysis. 


\section{Supplementary Material}

Refer to Web version on PubMed Central for supplementary material.

\section{Acknowledgments}

Funded by NIH NHLBI T32 HL007106-34.

This manuscript was prepared using FACTT Research Materials obtained from the NHLBI Biologic Specimen and Data Repository Information Coordinating Center and does not necessarily reflect the opinions or views of the ARDS Network or the NHLBI.

We thank the ARDSnet for providing us with the raw data of the FACTT study and for their insightful comments on this study.

We are also very grateful to Professor Joanne Garrett for her biostatistical input.

\section{Abbreviations}

APACHE Acute Physiology and Chronic Health Evaluation

ARDS acute respiratory distress syndrome

ARDSnet

CVP

FACTT

$\mathrm{FiO2}$

FRC

GEE

ICC

IRB

NIH

NHLBI

PAC

$\mathrm{PaO} 2$

PAOP

PEEP

PF
Acute Respiratory Distress Syndrome Clinical Trials Network

central venous pressure

Fluid and Catheter Treatment Trial

Fraction of inspired oxygen

functional residual capacity

generalized estimating equations

intra-class correlation

institutional review board

National Institute of Health

National Heart Lung and Blood Institute

pulmonary artery catheter

partial pressure of oxygen in the blood

pulmonary artery occlusion pressure also known as wedge pressure

positive end-expiratory pressure

$\mathrm{PaO} 2 / \mathrm{FiO} 2$ ratio

\section{References}

1. Rubenfeld GD, Caldwell E, Peabody E, et al. Incidence and outcomes of acute lung injury. N Engl J Med. 2005; 353:1685-1693. [PubMed: 16236739]

2. Bernard GR. Acute respiratory distress syndrome: a historical perspective. Am J Respir Crit Care Med. 2005; 172:798-806. [PubMed: 16020801]

3. Wiedemann HP, Wheeler AP, Bernard GR, et al. Comparison of two fluid-management strategies in acute lung injury. N Engl J Med. 2006; 354:2564-2575. [PubMed: 16714767]

4. Force ADT, Ranieri VM, Rubenfeld GD, et al. Acute respiratory distress syndrome: the Berlin Definition. JAMA. 2012; 307:2526-2533. [PubMed: 22797452] 
5. Needham DM, Colantuoni E, Mendez-Tellez PA, et al. Lung protective mechanical ventilation and two year survival in patients with acute lung injury: prospective cohort study. Bmj. 2012; 344:e2124. [PubMed: 22491953]

6. Tomashefski JF Jr, Davies P, Boggis C, et al. The pulmonary vascular lesions of the adult respiratory distress syndrome. Am J Pathol. 1983; 112:112-126. [PubMed: 6859225]

7. Slutsky AS. Neuromuscular Blocking Agents in ARDS. New Engl J Med. 2010; 363:1176-1180. [PubMed: 20843254]

8. Ventilation with lower tidal volumes as compared with traditional tidal volumes for acute lung injury and the acute respiratory distress syndrome. The Acute Respiratory Distress Syndrome Network. N Engl J Med. 2000; 342:1301-1308. [PubMed: 10793162]

9. Maggiore SM, Jonson B, Richard JC, et al. Alveolar derecruitment at decremental positive endexpiratory pressure levels in acute lung injury: comparison with the lower inflection point, oxygenation, and compliance. Am J Respir Crit Care Med. 2001; 164:795-801. [PubMed: 11549535]

10. Gernoth C, Wagner G, Pelosi P, et al. Respiratory and haemodynamic changes during decremental open lung positive end-expiratory pressure titration in patients with acute respiratory distress syndrome. Crit Care. 2009; 13

11. Malbouisson LM, Muller JC, Constantin JM, et al. Computed tomography assessment of positive end-expiratory pressure-induced alveolar recruitment in patients with acute respiratory distress syndrome. Am J Respir Crit Care Med. 2001; 163:1444-1450. [PubMed: 11371416]

12. Schmitt JM, Vieillard-Baron A, Augarde R, et al. Positive end-expiratory pressure titration in acute respiratory distress syndrome patients: impact on right ventricular outflow impedance evaluated by pulmonary artery Doppler flow velocity measurements. Crit Care Med. 2001; 29:1154-1158. [PubMed: 11395592]

13. Mekontso Dessap A, Boissier F, Leon R, et al. Prevalence and prognosis of shunting across patent foramen ovale during acute respiratory distress syndrome. Crit Care Med. 2010; 38:1786-1792. [PubMed: 20601861]

14. Mekontso Dessap A, Charron C, Devaquet J, et al. Impact of acute hypercapnia and augmented positive end-expiratory pressure on right ventricle function in severe acute respiratory distress syndrome. Intensive Care Med. 2009; 35:1850-1858. [PubMed: 19652953]

15. Fougeres E, Teboul JL, Richard C, et al. Hemodynamic impact of a positive endexpiratory pressure setting in acute respiratory distress syndrome: importance of the volume status. Crit Care Med. 2010; 38:802-807. [PubMed: 19926983]

16. Hess DR. How much PEEP? Do we need another meta-analysis? Respiratory care. 2011; 56:710_ 713. [PubMed: 21669106]

17. Malo J, Ali J, Wood LD. How does positive end-expiratory pressure reduce intrapulmonary shunt in canine pulmonary edema? Journal of applied physiology: respiratory, environmental and exercise physiology. 1984; 57:1002-1010.

18. Guerin C. The preventive role of higher PEEP in treating severely hypoxemic ARDS. Minerva anestesiologica. 2011; 77:835-845. [PubMed: 21730932]

19. Brower RG, Lanken PN, MacIntyre N, et al. Higher versus lower positive end-expiratory pressures in patients with the acute respiratory distress syndrome. N Engl J Med. 2004; 351:327-336. [PubMed: 15269312]

20. Meade MO, Cook DJ, Guyatt GH, et al. Ventilation strategy using low tidal volumes, recruitment maneuvers, and high positive end-expiratory pressure for acute lung injury and acute respiratory distress syndrome: a randomized controlled trial. JAMA. 2008; 299:637-645. [PubMed: 18270352]

21. Mercat A, Richard JC, Vielle B, et al. Positive end-expiratory pressure setting in adults with acute lung injury and acute respiratory distress syndrome: a randomized controlled trial. JAMA. 2008; 299:646-655. [PubMed: 18270353]

22. Briel M, Meade M, Mercat A, et al. Higher vs lower positive end-expiratory pressure in patients with acute lung injury and acute respiratory distress syndrome: systematic review and metaanalysis. JAMA. 2010; 303:865-873. [PubMed: 20197533] 
23. Wheeler AP, Bernard GR, Thompson BT, et al. Pulmonary-artery versus central venous catheter to guide treatment of acute lung injury. N Engl J Med. 2006; 354:2213-2224. [PubMed: 16714768]

24. Bernard GR, Artigas A, Brigham KL, et al. The American-European Consensus Conference on ARDS. Definitions, mechanisms, relevant outcomes, and clinical trial coordination. Am J Respir Crit Care Med. 1994; 149:818-824. [PubMed: 7509706]

25. Hanley JA, Negassa A, Edwardes MD, et al. Statistical analysis of correlated data using generalized estimating equations: an orientation. American journal of epidemiology. 2003; 157:364-375. [PubMed: 12578807]

26. Grissom CK, Morris AH, Lanken PN, et al. Association of physical examination with pulmonary artery catheter parameters in acute lung injury. Crit Care Med. 2009; 37:2720-2726. [PubMed: 19885995]

27. Huang CC, Fu JY, Hu HC, et al. Prediction of fluid responsiveness in acute respiratory distress syndrome patients ventilated with low tidal volume and high positive end-expiratory pressure. Crit Care Med. 2008; 36:2810-2816. [PubMed: 18766099]

28. Kumar A, Anel R, Bunnell E, et al. Pulmonary artery occlusion pressure and central venous pressure fail to predict ventricular filling volume, cardiac performance, or the response to volume infusion in normal subjects. Crit Care Med. 2004; 32:691-699. [PubMed: 15090949]

29. Talmor D, Sarge T, Malhotra A, et al. Mechanical ventilation guided by esophageal pressure in acute lung injury. N Engl J Med. 2008; 359:2095-2104. [PubMed: 19001507]

30. Puybasset L, Gusman P, Muller JC, et al. Regional distribution of gas and tissue in acute respiratory distress syndrome. III. Consequences for the effects of positive end-expiratory pressure. CT Scan ARDS Study Group. Adult Respiratory Distress Syndrome. Intensive Care Med. 2000; 26:1215-1227. [PubMed: 11089745]

31. Rouby JJ, Lu Q, Goldstein I. Selecting the right level of positive end-expiratory pressure in patients with acute respiratory distress syndrome. Am J Respir Crit Care Med. 2002; 165:11821186. [PubMed: 11956065]

32. Luecke T, Pelosi P. Clinical review: Positive end-expiratory pressure and cardiac output. Crit Care. 2005; 9:607-621. [PubMed: 16356246]

33. Qvist J, Pontoppidan H, Wilson RS, et al. Hemodynamic responses to mechanical ventilation with PEEP: the effect of hypervolemia. Anesthesiology. 1975; 42:45-55. [PubMed: 234210]

34. Gattinoni L, Pesenti A, Baglioni S, et al. Inflammatory pulmonary edema and positive endexpiratory pressure: correlations between imaging and physiologic studies. Journal of thoracic imaging. 1988; 3:59-64. [PubMed: 3292784]

35. Monnet X, Bleibtreu A, Ferre A, et al. Passive leg-raising and end-expiratory occlusion tests perform better than pulse pressure variation in patients with low respiratory system compliance. Crit Care Med. 2012; 40:152-157. [PubMed: 21926581]

36. Jardin F, Vieillard-Baron A. Is there a safe plateau pressure in ARDS? The right heart only knows. Intensive Care Med. 2007; 33:444-447. [PubMed: 17268795]

37. Fares WH, Blanchard SK, Stouffer GA, et al. Thermodilution and Fick cardiac outputs differ: Impact on pulmonary hypertension evaluation. Canadian respiratory journal : journal of the Canadian Thoracic Society. 2012; 19:261-266. [PubMed: 22891186] 


\section{2,910 simultaneous PEEP and cardiac index measurements / observations done in the first 3 days of the study

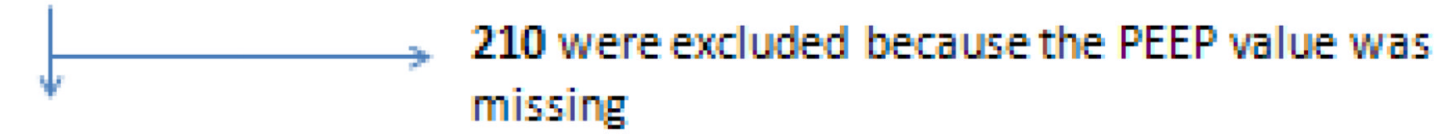 \\ 2,700 \\ 103 were excluded because the FiO2 value was missing}

2,597

$$
\downarrow
$$

244 were excluded because PaO2 was missing.

\section{2,353}

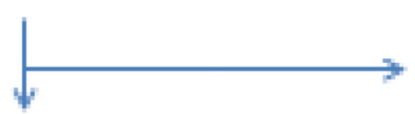

\section{1,205 were excluded because $\mathrm{Cl}$ was missing.}

\section{1,148}

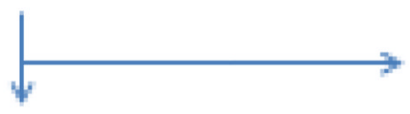

\section{5 were excluded because they were on a vasopressor or an inotrope}

\section{Final study population of 833 simultaneous PEEP and}

\section{cardiac index measurements}

Fig. 1.

Reasons for ( \& numbers of) exclusions from the final analysis. PEEP: positive endexpiratory pressure. Observations with missing PEEP were excluded because PEEP is the primary exposure variable. Observations with missing cardiac index were excluded because cardiac index is the primary outcome variable. Observations with missing FiO2 or $\mathrm{PaO} 2$ were excluded because PF ratio could not be calculated for these observations, so a diagnosis of ARDS could not be determined. 


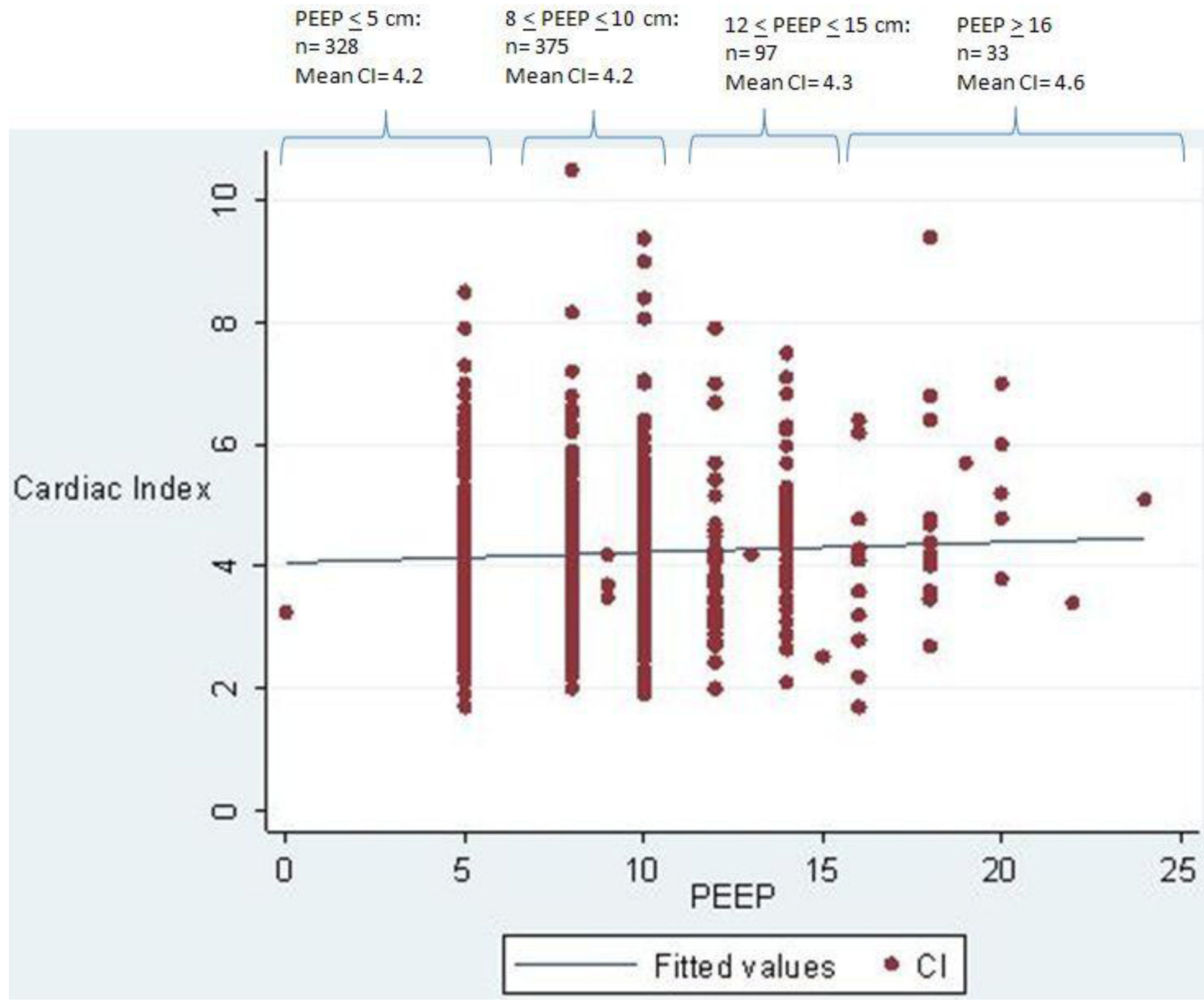

Fig. 2.

Unadjusted scatterplot of Positive End-Expiratory Pressure (PEEP) and cardiac index (CI) $(n=833)$. Correlation coefficient $(r)=0.05 ; p=0.17$. Numbers of observations within 4 different PEEP categories are also noted at the top of the figure, with the mean CI for each category. 


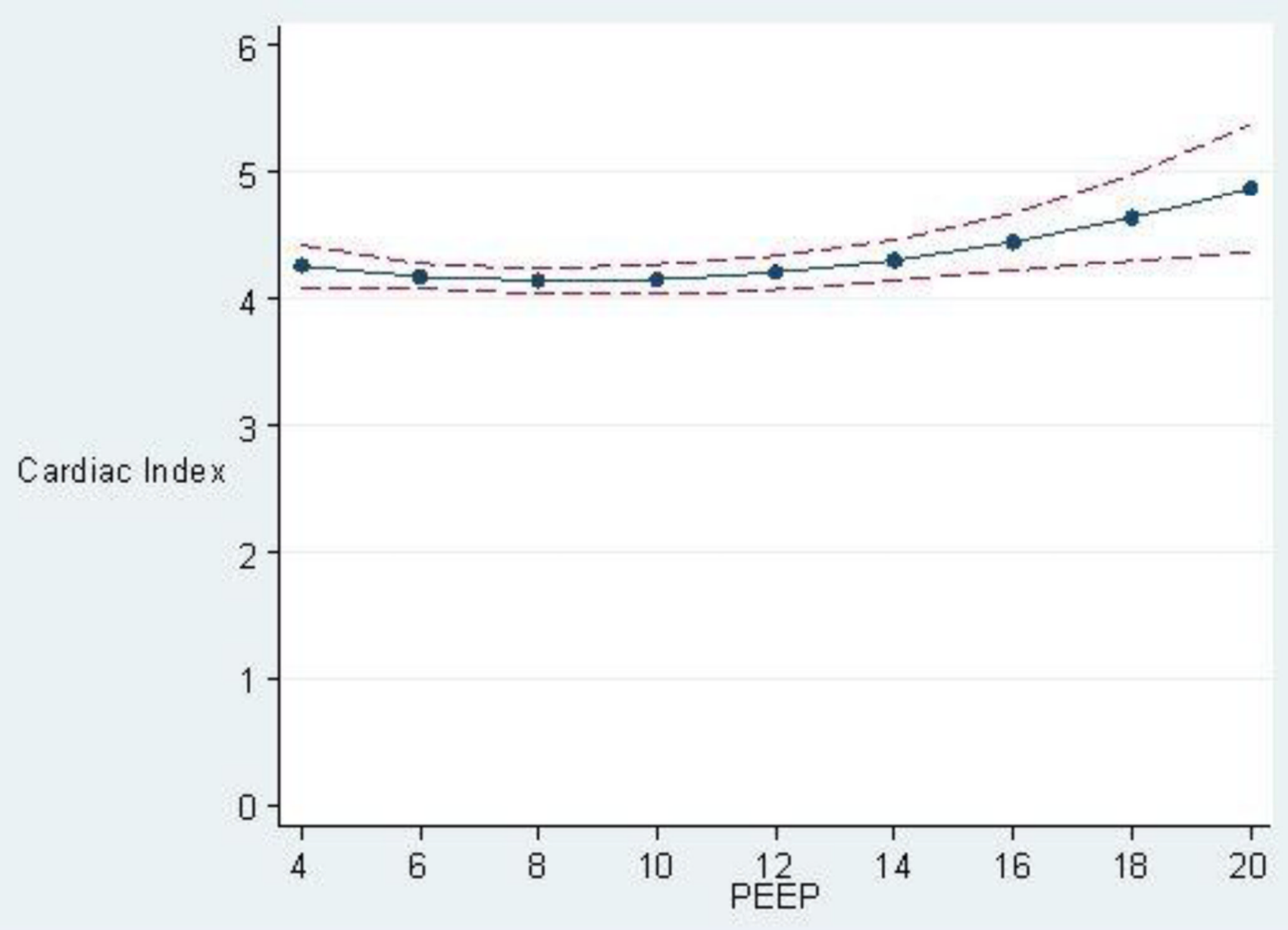

Fig. 3.

Predicted Cardiac Index (in liters / minute / meter $^{2}$ ) with 95\% confidence interval, based on the PEEP level in cm adjusted for APACHE III score, age, FACTT study fluids arm (liberal versus conservative), and sepsis (present versus absent). $\mathrm{p}=0.71$. 


\section{Table 1}

Patients Characteristics.

\begin{tabular}{|c|c|c|}
\hline & \multicolumn{2}{|l|}{ Mean $( \pm$ SD) or $\%$} \\
\hline Age (years) & $49 \pm 15$ & \\
\hline Female Gender & $45 \%$ & \\
\hline \multirow{2}{*}{ Ethnicity } & Caucasian: & $64 \%$ \\
\hline & African American: & $24 \%$ \\
\hline APACHE III score & \multicolumn{2}{|l|}{$90 \pm 30$} \\
\hline BMI & \multicolumn{2}{|l|}{$29.6 \pm 6.9$} \\
\hline Medical Hospital Admission Type & \multicolumn{2}{|c|}{$80 \%$ had a Medical hospital admission type (e.g. rather than Surgical) } \\
\hline Direct Admit from the ED & \multicolumn{2}{|l|}{$36 \%$} \\
\hline \multicolumn{3}{|c|}{ ARDS Etiology } \\
\hline & Primary Etiology of ARDS & Secondary Contributing ARDS Factor \\
\hline Pneumonia & $48 \%$ & $16 \%$ \\
\hline Sepsis & $20 \%$ & $20 \%$ \\
\hline Aspiration & $15 \%$ & $6 \%$ \\
\hline Trauma & $9 \%$ & $<1 \%$ \\
\hline Other Lung Injury & $7 \%$ & $2 \%$ \\
\hline Multiple Transfusions & $<1 \%$ & $2 \%$ \\
\hline \multicolumn{3}{|c|}{ Comorbidities } \\
\hline Hypertension & $30 \%$ & \\
\hline Vasopressors need in the last 24 hours prior to randomization & $28 \%$ & \\
\hline Diabetes Mellitus & $19 \%$ & \\
\hline Alcohol Use & $10 \%$ & \\
\hline Chronic Pulmonary Disease & $8 \%$ & \\
\hline Immunosuppressed in the Last 6 months & $8 \%$ & \\
\hline Prior MI & $6 \%$ & \\
\hline AIDS & $6 \%$ & \\
\hline Peripheral Vascular Disease & $5 \%$ & \\
\hline Congestive Heart Failure & $4 \%$ & \\
\hline Prior Stroke & $4 \%$ & \\
\hline
\end{tabular}

Demographics and characteristics of the study population ( $\mathrm{N}=367$ patients).

SD: Standard Deviation. APACHE: Acute Physiology and Chronic Health Evaluation. BMI: Body Mass Index. ED: Emergency Department. ARDS: Acute Respiratory Distress Syndrome. MI: Myocardial Infarction. AIDS: Acquired Immune Deficiency Syndrome. 
Table 2

Observations characteristics stratified by PEEP groups

\begin{tabular}{lcccc}
\hline Characteristic & PEEP $\leq \mathbf{5 c m}$ & $\mathbf{8} \leq$ PEEP $\mathbf{4 0 c m}$ & $\mathbf{1 2} \leq$ PEEP $\leq \mathbf{1 5} \mathbf{c m}$ & PEEP $\geq \mathbf{1 6 c m}$ \\
\hline \# of observations & 328 & 375 & 97 & 33 \\
Cardiac index ${ }^{*}(1 / \mathrm{min} / \mathrm{m} 2)$ & $4.2 \pm 1.1$ & $4.2 \pm 1.3$ & $4.3 \pm 1.2$ & $4.6 \pm 1.5$ \\
CVP (cm water) & $10 \pm 4$ & $12 \pm 4$ & $13 \pm 5$ & $15 \pm 5$ \\
PAOP (cm water) & $13 \pm 5$ & $15 \pm 5$ & $16 \pm 5$ & $18 \pm 5$ \\
PaO2/FiO2 ratio & $224 \pm 75$ & $166 \pm 67$ & $112 \pm 49$ & $81 \pm 25$ \\
Plateau pressure (cm water) & $20 \pm 5$ & $24 \pm 5$ & $29 \pm 6$ & $33 \pm 8$ \\
Lung Compliance (ml / cm water) & $29 \pm 13$ & $31 \pm 16$ & $30 \pm 21$ & $31 \pm 34$ \\
FACTT study arm, \% in liberal fluid arm & $47 \%$ & $50 \%$ & $64 \%$ & $70 \%$ \\
\hline
\end{tabular}

Observations characteristics stratified by PEEP groups ( $N=833$ dual measurements). Please note that there were no observations in the study group that had PEEP values of $6 \mathrm{~cm}, 7 \mathrm{~cm}$, or $11 \mathrm{~cm}$, and there was only one observation with a PEEP of $13 \mathrm{~cm}$ and another observation with a PEEP of 15 $\mathrm{cm}$.

PEEP: positive end-expiratory pressure. CVP: central venous pressure. PAOP: pulmonary artery occlusion pressure, also known as wedge pressure.

* The differences in cardiac index between the 4 PEEP categories were not statistically significant when adjusted for in the multiple linear regression model $(\mathrm{p}=0.5)$ 